\title{
Kopf-Hals-Tumoren
}

\section{Interdisziplinäre Zusammenarbeit}

Die Bedeutung der Therapie mit Cetuximab bei rezidivierten und/oder metastasierten Kopf-Hals-Tumoren spiegelt sich in den aktuellen Leitlinien der ESMO (European Society for Medical Oncology) wider. Um jedoch ein Optimum für die Patienten zu erreichen, müssen Onkologen, Hals-Nasen-Ohren-Ärzte und Strahlentherapeuten künftig noch enger zusammenarbeiten.

Die Therapie fortgeschrittener Kopf-HalsTumoren stellt eine interdisziplinäre Herausforderung dar, die regionale und multizentrische Absprachen erfordert, so Susanne Staar, Bremen. Ziel muss es sein, durch optimale therapeutische Kombinationen einen langfristigen maximalen Organ- und Funktionserhalt zu erreichen. Dass ein derartiges Vorgehen Erfolg hat, lässt sich an den im Vergleich zu Deutschland besseren Überlebensraten in den Niederlanden ablesen. Hier wird in den etablierten Krebszentren bereits eng zusammengearbeitet.

Kopf-Hals-Tumoren sind weltweit die sechsthäufigste Krebserkrankung. In Europa gibt es jährlich mehr als 140.000 Neuerkrankungen und fast 70.000 Todesfälle. Zu den Hauptrisikofaktoren gehören Alkoholkonsum und Rauchen. Mehr als 90\% aller Kopf-Hals-Tumoren sind Plattenepithelkarzinome, die fast alle den epidermalen Wachstumsfaktorrezeptor (EGFR), die Zielstruktur des therapeutischen Antikörpers Cetuximab, an der Zelloberfläche exprimieren.

\section{Empfehlung für die First-line-Therapie mit Cetuximab}

Cetuximab $\left(\right.$ Erbitux $\left.^{\oplus}\right)$ ist seit November 2008 in Kombination mit einer platinbasierten Standardchemotherapie zugelassen für die First-line-Therapie bei Patienten mit rezidivierten und/oder metastasierten Plattenepithelkarzinomen des Kopfes und Halses (Squamous Cell Carcinoma of the Head and Neck [SCCHN]). Für diese Indikation hat die Kombination als einzige Therapieoption die Empfehlung mit dem Evidenzgrad A (aktuelle ESMOLeitlinien) und gilt deshalb als neuer Therapiestandard.

Die Zulassung von Cetuximab für die Behandlung von lokal fortgeschrittenen SCCHN in Kombination mit einer Strahlentherapie liegt seit 2006 vor. Aktuelle 5-Jahres-Überlebensdaten von Bonner et al. (Bonner JA et al., 2010, Lancet Oncol 11: 21-28) bestätigen die Vorteile der adjuvanten Cetuximab-Gabe zusätzlich zu einer Strahlentherapie (Abb.).

In interdisziplinären Projekten sollen die Möglichkeiten evaluiert werden, die

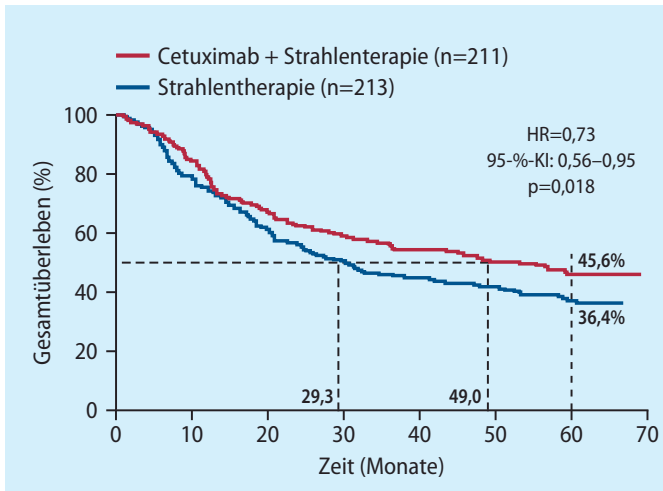

95-\%-Kl=95-\%-Konfidenzintervall; HR=Hazard-Ratio

nach Bonner JA et al., 2010, Lancet Oncol 11: 21-28

Gesamtüberleben von Patienten mit lokal fortgeschrittenen Kopf-Hals-Tumoren: Hinzunahme von Cetuximab zur Strahlentherapie versus Strahlentherapie allein

Lebensqualität der Patienten weiter zu verbessern. Ebenso im Fokus: Die molekularbiologische Tumortypisierung, die eine wichtige Rolle bei der Optimierung der Therapiestrategien spielen wird, wie Ulrich Keilholz, Berlin, betonte. UF

Quelle: Veranstaltung der Merck Serono $\mathrm{GmbH}$

\section{Pankreaskarzinom}

\section{Management von Hautreaktionen unter Erlotinib}

Eine spezifische medikamentöse Prophylaxe von Hautreaktionen bei der Therapie mit Substanzen, die die Signaltransduktion des epidermalen Wachstumsfaktorrezeptors (EGFR) hemmen, wird derzeit nicht empfohlen. Wichtig sind laut Ralf Gutzmer, Hannover, die Information der Patienten über diese Nebenwirkung und eine rückfettende Hautpflege. Die Therapie sollte beim ersten Auftreten von Hautreaktionen vom primär behandelnden Arzt begonnen werden; sie richtet sich nach dem klinischen Bild und Schweregrad der Hautreaktion.

Hautreaktionen sind eine häufige Nebenwirkung von Substanzen, die die EGFRSignaltransduktion hemmen. Durch die Inhibition der EGFR-Signalkaskade nicht nur im Tumorgewebe, sondern auch in der Haut werden laut Gutzmer die Proliferation und Differenzierung der Keratinozyten gehemmt und die immunologische Homöostase verändert: Die Haut reagiert schneller und leichter auf Entzündungsreize.

In Studien, wie beispielsweise auch in der Studie von Moore et al. zur Therapie mit dem EGFR-Tyrosinkinasehemmer Erlotinib $\left(\operatorname{Tarceva}^{\oplus}\right)$ bei Patienten mit Pankreaskarzinom, konnte eine Korrelation zwischen dem Auftreten von Hautreaktionen und der Wirksamkeit der Therapie gezeigt werden: Bei Patienten mit einer Hautreaktion vom Schweregrad $>1$ wurde im Vergleich zu Patienten ohne Hautreaktion ein signifikant verlängertes Gesamtüberleben beobachtet (median 10,5 Monate vs. 5,3 Monate; $p<0,0001$; Moore MJ et al., 2007, J Clin Oncol 25: 1960-1966). Laut Gutzmer ist der biologische Zusammenhang dafür bislang unklar. 